[6;93] mo, average disease activity (DAS28) 5,2 $\pm 1,7$. CKD was defined as the presence of markers of renal impairment (proteinuria $\geq 1+$, hematuria $\geq 2+$ or leucocyturia $\geq 2+$ ) and/or eGFR $<60 \mathrm{ml} / \mathrm{min} / 1,73 \mathrm{~m}^{2}$ (using CKD-EPI formula) persistent beyond 3 months. The extent of multi-morbid environment was rated using Cumulative Illness Rating Score (CIRS) [2]. CIRS was calculated using the 5 score scale (0-4) to assess 14 major organ systems of the body (0-56).

Results: The prevalence of CKD was $82,3 \%(172)$, and $30,1 \%(63)$ had eGFR $<60$ $\mathrm{ml} / \mathrm{min} / 1,73 \mathrm{~m}$, and $50,2 \%$ pts had CKD stage 2. The range of proteinuria was $12,4 \%$. In group of pts with CKD 3-5 stage the prevalence of arterial hypertension (AH) was $84 \%$, ischemic heart disease (IHD) - $63,5 \%$, diabetes militants 2 $22 \%$, oncology $12,7 \%$. Mean CIRS value in RA pts was 15 [10;19] scores. The eGFR was independent of the RA duration $(r=-0,01, p=0,86)$ and RA activity $(r=0,09, p=0,17)$. There was found the strongest correlation of eGFR with CIRS $(r=-0,61, p<0,01)$, age $(r=0,58 ; p<0,05)$, AH $(r=-0,34, p<0,01)$, IHD $(r=-0,28, p<0,05)$, BMI $(r=-0,17 ; r=0,35)$, uric acid levels $(r=-0,37)$, as well as with hemoglobin level $(r=0,19)$ and $\mathrm{HAQ}$ scores $(r=0,31 ; r=0,32)$. No correlation was found between eGFR and oncology, gender, VAS score, and ESR, CRP, cholesterol $(p>0,05)$.

Conclusions: CKD is a serious condition associated with premature mortality, decreased quality of life, and increased health-care expenditures, but it doesn't consider as comorbid disorder of RA. The CKD prevalence is greater among aged RA pts than in general population and it has a stronger correlation with multimorbid burden than traditional factors. Compromised renal function, apart from the age, is of dominant importance in pts management issues, limiting the range of available effective therapies, thus, aggravating RA natural course on one side, and inducing and catalyzing the severity of dysmetabolic syndrome, $\mathrm{AH}$ and anemia - on the other.

References:

[1] Hudon C, Fortin M, Vanasse A. Cumulative illness rating scale was a reliable and valid index in a family practice context. J Clin Epidemiol 2005;58:603-8. [2] Matsushita K, van der Velde M, Astor BC et al. Lancet 2010;375:2073-81. Acknowledgements: None.

Disclosure of Interest: None declared

DOI: 10.1136/annrheumdis-2017-eular.6839

\section{THU0121 ULTRASOUND IN THE ASSESSMENT OF CARPAL TUNNEL SYNDROME IN PATIENTS WITH RHEUMATOID ARTHRITIS}

A. Di Matteo, E. Filippucci, G. Smerilli, A. Draghessi, S. Gasparini, A. Incorvaia, M. Di Carlo, W. Grassi. Clinica Reumatologica, Università Politecnica delle Marche, Jesi $(A N)$, Italy

Background: Carpal tunnel syndrome (CTS) is one of the most frequent extraarticular manifestations of rheumatoid arthritis (RA). Ultrasound (US) has proven to represent a reliable tool for the diagnosis of CTS [1]. However, its role in the diagnosis of CTS in patients with RA has been poorly investigated.

Objectives: The aim of this study is to evaluate the US findings at carpal tunnel level in a cohort of patients with RA, focusing on those with a clinical diagnosis of CTS.

Methods: Patients with RA fulfilling the ACR/EULAR 2010 classification criteria were consecutively enrolled. The diagnosis of CTS was made according to the American Academy of Neurology practice parameter for CTS [2]. The MSUS assessment was carried out using a MyLab Twice (Esaote SPA) US system working with a 18-22 MHz linear probe. The power Doppler (PD) frequency was set between 7.5 and $11.3 \mathrm{MHz}$. The following grey scale (GS) US parameters were assessed at the carpal tunnel level: cross-sectional area (CSA) of the median nerve at the carpal tunnel inlet (at the level of the pisiform and scaphoid bones), presence of flexor tenosynovitis and palmar radio-carpal synovitis (both in GS and PD), presence of crystal macro-aggregates and marked bone profile irregularities. The median nerve was considered enlarged if its CSA was more than $12 \mathrm{~mm}^{2}$. We evaluated the presence of intra-neural PD signals at the carpal inlet and scored its entity $(0=$ no PD signal, $1=$ one single vessel within median nerve, $2=$ two or three single or two confluent vessels and $3=$ more than three single or more than two confluent vessels). PD was considered "positive" if grade 1 or more was found.

Results: We included 40 RA patients. CTS was diagnosed in 19 out of 80 wrists $(23.8 \%)$ and in 13 out of 40 RA patients $(32.5 \%)$. Enlarged median nerve was found in 3 out of 19 wrists with CTS (15.8\%) and in 6 out of 61 wrists without CTS $(9.8 \%)$. Flexor tenosynovitis was found in 7 out of 19 wrists with CTS $(36.8 \%)$ and in 5 out of 61 wrists without CTS $(8.2 \%)$. Palmar radio-carpal synovitis was found in 2 out of 19 wrists with CTS (10.5\%) and in 3 out of 61 wrists without CTS (4.9\%). Crystal macro-aggregates were not detected in any of the scanned wrists. Marked bone profile irregularities were found in 2 out of 19 wrists with CTS $(10.5 \%)$ and in 14 out of 61 wrists without CTS $(23 \%)$. Positive intra-neural PD was found in 9 out of 19 wrists with CTS (47.4\%) and in 9 out of 61 wrists without CTS $(14.7 \%)$

Conclusions: These preliminary results suggest that MSUS could be a useful tool in the diagnosis of CTS also in patients with RA. Intra-neural PD and flexor tenosynovitis were the most frequently MSUS abnormalities detected in RA patients with CTS. The inflammatory involvement of the tendinous and joint structures which are part of the carpal tunnel could lead to median nerve compression and CTS symptoms and should be considered in the MSUS assessment of CTS.

\section{References:}

[1] McDonagh C, Alexander M, Kane D. The role of ultrasound in the diagnosis and managment of carpal tunnel sindrome: a new paradigme. Rheumatology (Oxford). 2015 Jan;54(1):9-19.doi: 10.1093/rheumatology/keu275.

[2] Practice parameters for carpal tunnel syndrome (summary statement). Report of the quality standards subcommittee of the American Academy of Neurology. Neurology 1993;43:2406-9.

Disclosure of Interest: None declared

DOI: 10.1136/annrheumdis-2017-eular.4448

\section{THU0122 QUANTITATIVE ASSESSMENT AND ANALYSIS OF HAND MUSCLE VOLUME}

A. Friedberger ${ }^{1}$, C. Figueiredo ${ }^{2}$, O. Museyko ${ }^{1}$, A. Grimm ${ }^{1}$, I. D'Oliveira ${ }^{2}$, T. Bäuerle ${ }^{3}$, J. Rech ${ }^{2}$, O. Chaudry ${ }^{1}$, M. Uder ${ }^{3}$, G. Schett ${ }^{2}$, K. Engelke ${ }^{1}$.' ${ }^{1}$ Institute of Medical Physics, University Erlangen-Nuremberg; ${ }^{2}$ Department of Medicine 3, University Erlangen-Nuremberg; ${ }^{3}$ Radiological Institute, University Hospital Erlangen-Nuremberg, Erlangen, Germany

Background: Rheumatoid arthritis (RA) is a chronic inflammatory disease characterized by bone, cartilage and muscle loss. While bone and cartilage damage have been extensively studied in the past, the effects of RA on volume and composition of hand muscles have not yet been studied. This situation is surprising, since visible hand muscle atrophy is a hallmark of RA and quantification of muscle composition is of growing interest.

Objectives: Quantitative assessment of hand muscle volume and fat using MRI Methods: A random forest based method was used to segment hand muscle in T1 weighted MR scans of 76 RA patients (37 males, 26-87 years, mean 61 years). The segmentation procedure is fully automated but allows for manual corrections of wrongly segmented areas. Multimodal registration of the muscle segmentation masks to MR Dixon Fat Fraction images was used for fat quantification.

Outcome parameters were absolute hand and muscle volume $\left(V_{H}^{\text {abs }}\right.$ resp. $\left.V_{M}^{\text {abs }}\right)$, relative muscle volume $\mathrm{V}_{\mathrm{M}}^{\text {rel }}=\mathrm{V}_{\mathrm{M}}^{\text {abs }} / \mathrm{V}_{\mathrm{H}}^{\text {abs }}$ and absolute and relative fat content $\left(\mathrm{V}_{\mathrm{F}}^{\text {abs }}\right.$ resp. $V_{F}^{r e l}$ ).

Student's t-tests were performed for gender discrimination. Linear regression was used to model age dependency. Further a multivariate regression was used to model dependence on predictors BMI, RA disease duration, DMARD treatment duration, HAQ, DAS28, RF, ESR and CRP after age adjustment. Dixon sequences were not available for all patients, therefore fat analysis could only be done in 17 females.

Results: $V_{M}^{a b s}$ in males was significantly higher than in females $(p<0.001$, means: $63 \mathrm{~cm}^{3}$ vs. $\left.29 \mathrm{~cm}^{3}\right)$. Similar results were observed for $V_{H}^{\text {abs }}(p<0.001$, means: 243 $\mathrm{cm}^{3}$ vs. $163 \mathrm{~cm}^{3}$ ) and $V_{M}^{\text {rel }}(p=0.02$, means: 0.256 vs. 0.239 ).

The table show the results of the linear regression analysis for significant predictors of males and females, respectively. The figure shows age dependence of $\mathrm{V}_{\mathrm{M}}^{\mathrm{abs}}$ and $\mathrm{V}_{\mathrm{H}}^{\mathrm{abs}}$.

\begin{tabular}{cccc}
\hline & Age & BMl (age adj.) & Disease duration (age adj.) \\
\hline Males & & & \\
$V_{M}^{\text {abs }}$ & $p<0.001, R^{2}=0.25$ & $p<0.001, R^{2}=0.61$ & $n s$. \\
$V_{H}^{\text {abs }}$ & $p=0.03, R^{2}=0.13$ & $p<0.001, R^{2}=0.50$ & $n s$. \\
$V_{M}^{\text {rel }}$ & $p<0.001, R^{2}=0.30$ & $p=0.04, R^{2}=0.35$ & $p=0.02, R^{2}=0.36$ \\
Females & $n s$. & $n s$. & $p=0.07, R^{2}=0.16$ \\
$V_{M}^{\text {abs }}$ & $n$ & $n s$. & $n s$. \\
$V_{H}^{\text {abs }}$ & $p<0.001, R^{2}=0.24$ & $n s$. & $p=0.06, R 2=0.12$ \\
$V_{M}^{\text {rel }}$ & $n s$. & $n$ & \\
\hline
\end{tabular}

$V_{F}^{a b s}$ and $V_{F}^{\text {rel }}$ showed no dependence on age or disease duration. $V_{F}^{\text {abs }}$ but not $V_{F}^{\text {rel }}$ was highly correlated with $V_{M}^{\text {abs }}$ and $V_{H}^{\text {abs }}$ (both: $p<0.001, R^{2}=0.75$ ). $V_{F}^{\text {rel }}$ was positively correlated with $\mathrm{BMI}\left(\mathrm{p}=0.03, \mathrm{R}^{2}=0.32\right)$, a weaker but not significant effect was observed for $\mathrm{V}_{\mathrm{F}}^{\text {abs }}$.
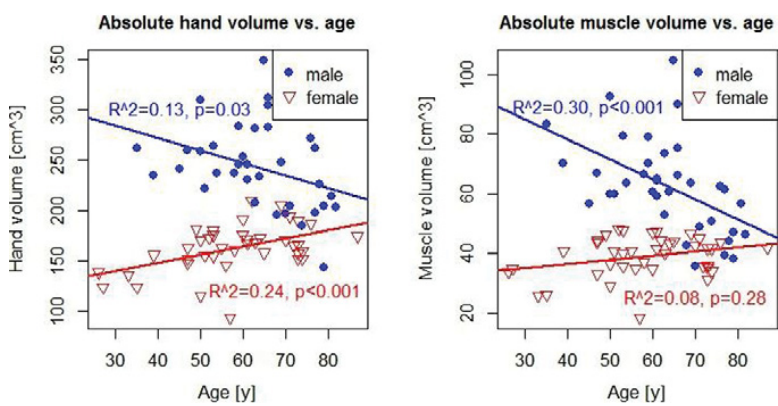

Conclusions: Results for muscle volume were highly sex specific. Males showed higher $\mathrm{V}_{\mathrm{M}}^{\text {abs }}, \mathrm{V}_{\mathrm{H}}^{\text {abs }}$ and $\mathrm{V}_{\mathrm{M}}^{\text {rel }}$. Nevertheless $\mathrm{V}_{\mathrm{M}}^{\text {abs }}$ and $\mathrm{V}_{\mathrm{H}}^{\text {abs }}$ in males showed significant decrease with age while the opposite but weaker effects were observed in females. Relative fat volume depends on BMI but is independent of muscle volume.

Disclosure of Interest: None declared

DOI: 10.1136/annrheumdis-2017-eular.6084 